three patients who needed embolisation to control bleeding (two patients in this group died within $24 \mathrm{~h}$ ).

Conclusion Hospital readmission rates after hepatic and pancreatic resection are acceptable. However, readmitted patients have a very high morbidity, often requiring urgent intervention only available at a specialist centre. Efficient communication and rapid transfer of patients to a centre with the available expertise is vital to prevent delayed deaths after major surgery.

Competing interests None declared.

\section{PTU-078 DELAYS IN THE DIAGNOSIS AND MANAGEMENT OF PATIENTS WITH SUSPECTED SPHINCTER OF ODDI DYSUNCTION}

doi:10.1136/gutjnl-2012-302514c.78

${ }^{1} \mathrm{E} J$ Lunn, ${ }^{*}{ }^{2} \mathrm{~F}$ Gohar, ${ }^{3} \mathrm{P}$ Basumani, ${ }^{4} \mathrm{M}$ Karajeh, ${ }^{2} \mathrm{~K}$ Kapur. ${ }^{1}$ Gastroenterology, Barnsley, Sheffield, UK; ${ }^{2}$ Gastroenterology, Barnsley, UK; ${ }^{3}$ Gastroenterology, Rotherham, UK; ${ }^{4}$ Gastroenterology, Sheffield Teaching Hospitals, Sheffield, UK

Introduction Sphincter of oddi dysfunction (SOD) is difficult to diagnose and treat. Biliary manometry is considered to be the gold standard for diagnosing SOD but is not widely available. A significant number of patients, that we think are likely to have sphincter oddi dysfunction, present to our hospitals with recurrent upper abdominal pain. They have multiple investigations, recurrent $\mathrm{A}+\mathrm{E}$ attendances and repeated admissions without a clear diagnosis or definitive treatment. Our aim was to identify this patient group in order to streamline their investigations and allow definitive treatment at an early stage, prevent readmission and save resources.

Methods A retrospective case note review of patients across three hospitals in South Yorkshire, in whom the final diagnosis was SOD based on their clinical presentation and investigations.

Results We reviewed 40 case notes in total. $88 \%$ of patients were female with a median patient age of 40 (18-75 years) Patients on average presented to $A+E 6$ times (0-50), median number of inpatient admissions was $4(0-20)$ with additional outpatient clinic appointments. $70 \%(28 / 40)$ of patients have previously undergone cholecystectomy, with $100 \%$ continuing to have similar pain to that prior to surgery. The most common provisional diagnosis at presentation was bile duct stones (38\%). Median duration of symptoms was 3 years (range 5 months-23 years). 35\% (14/40) of patients initially presented to the surgeons. $100 \%$ of patient had abdominal USS $(1-5)$ and $63 \%$ had undergone at least one OGD. All patients had a MRCP (range 1-4), 17 (43\%) patients had a CT abdomen (0-4) and $12(30 \%)$ patients underwent a HIDA scan. Patients were categorised into SOD type $1(22 \%)$, type $2(56 \%)$ or type $3(22 \%)$ on their clinical presentation and investigations. $28 \%(7 / 40)$ of patient had a trial of Botox, $48 \%(19 / 40)$ underwent ERCP and biliary sphincterotomy with 53\% (10/19) having symptomatic improvement. The remainder were managed on medial therapy.

Conclusion There is a significant group of patients, who have recurrent abdominal pain, recurrent admissions, undergo multiple investigations and trials of medical therapy without a definitive diagnosis being made. In addition, these patients are often subjected to invasive interventions such as ERCP and sphincterotomy with the potential risk of serious complications. This audit highlights the need for a designated service to streamline work-up and management of these patients: both to reduce cost and to improve outcomes.

Competing interests None declared.

\section{PTU-079 PROSPECTIVE AUDIT OF READMISSION FOLLOWING EMERGENCY AND ELECTIVE CHOLECYSTECTOMY IN A SINGLE HEALTH BOARD}

doi:10.1136/gutjnl-2012-302514c.79

${ }^{1} \mathrm{E} J$ Barron, ${ }^{*}{ }^{1} \mathrm{~J}$ P Blackmur, ${ }^{2} \mathrm{R}$ McMurray, ${ }^{1} \mathrm{E}$ M Harrison, ${ }^{3} \mathrm{~T}$ E Gillies, ${ }^{1} \mathrm{~J}$ Garden. ${ }^{1}$ Clinical and Surgical Sciences (Surgery), Edinburgh, UK; ${ }^{2}$ University of Edinburgh, Edinburgh, UK; ${ }^{3}$ General surgery, Royal Infirmary Edinburgh, Edinburgh, UK

Introduction Surgical outcome indicators, such as hospital stay, readmission and mortality rates, are increasingly being used to assess and compare hospital board performance and considerable variation exists between hospitals specifically in readmission rates. The aim of the study was to determine true readmission rates following cholecystectomy in a single large volume centre to determine whether readmission was potentially preventable.

Methods All patients readmitted to one large teaching hospital surgical service within 28 days following elective or emergency cholecystectomy from September 2010 to June 2011 were audited prospectively.

Results Of 979 cholecystectomies performed during the period, 57 $(5.8 \%)$ patients were readmitted. 38 of the $57(67 \%)$ readmissions followed emergency-admission with symptomatic gallstone disease and 51 of these $(89.5 \%)$ had undergone a laparoscopic approach. $34 / 979$ (3.5\%) were considered to be secondary to demonstrable complications of surgery with the most common cause being retained stones (11). No patient presented with bile duct injury, and there were no deaths. Only 14 of the readmitted patients $(25 \%)$ required intervention: one required sub-phrenic abscess drainage, nine endoscopic-retrograde-cholangiopancreatography and sphincterotomy (ERCP), two completion cholecystectomy, one laparoscopic assessment following ERCP for bile leak and one underwent hepatico-jejunostomy for definitive management of an irretrievable retained stone following ERCP and laparoscopic bile duct exploration. Of those readmitted, the most common cause of presentation was non-specific abdominal pain (15 (26.3\%)) with no cause found.

Conclusion Readmission rate in this large volume centre was low. Most patients readmitted following cholecystectomy have demonstrable surgically related complications but few require definitive surgical management. Further work is being conducted to define potential predictive factors for readmission.

Competing interests None declared.

\section{PTU-080 POLYUNSATURATED PHOSPHATIDILCHOLINE AND SIBUTRAMIN DECREASE THE LIVER FIBROSIS PROGRESS IN PATIENTS WITH NON-ALCOHOLIC LIVER DISEASE}

doi:10.1136/gutjnl-2012-302514c.80

${ }^{1,2}{ }^{2}$ Sas, * $1 V$ Grinevich, ' U Kravchuk. 'Department of Therapy Postgraduate Education, St.-Petersburg, Russian Federation; ${ }^{2}$ Military-Medical Academy, St.-Petersburg, Russian Federation

Introduction From 2009 to 2011 in our randomised prospective, blinded clinical trial we studied the effect of polyunsaturated phosphatidylcholine-PUPC (Essentiale ${ }^{\circledR}$ forte N, A. Nattermann \& Cie.GmbH) and Sibutramin in patients with obesity (BMI $30-35 \mathrm{~kg} / \mathrm{m}^{2}$ ). We studied 80 patients with obesity mean age of $38 \pm 7$ years and 40 were males. High resolution B mode ultrasonography was carried out twice for screening NAFL patients and after 6 months treatment.

Methods Liver function markers ALT, AST and GGT were measured twice, before and after 6 months treatment. All patients followed the basic treatment scheme included dietary and physical regimen. 